\title{
SOP Therapiezieländerung
}

Björn Ellger, Corinna Kelbel, Julian Bösel

\begin{abstract}
In dieser Rubrik stellen wir Standard Operating Procedures (SOPs) für häufige, intensivmedizinisch relevante Prozesse vor. Die Form ist eher im Sinne einer Schablone zu verstehen als - durchaus subjektiv gefärbte - Anregung, eigene, auf lokale Gegebenheiten adaptierte stationsinterne SOPs zu entwerfen und zu implementieren.
\end{abstract}

\section{Einleitung zur SOP Therapiezieländerung}

Zu dem Thema existiert ein ausführliches Positionspapier der DIVI, dessen Lektüre sehr zu empfehlen ist [1].

Jeder Patient, der auf die Intensivstation aufgenommen wird, ist zunächst mit einem kurativen Ansatz in der Hoffnung auf eine Restitutio ad integrum zu behandeln. Auch Patienten mit einer akut lebenslimitierenden Erkrankung können auf die Intensivstation aufgenommen werden, um akute Probleme zu behandeln (z. B. eine Pneumonie bei einem Plasmozytom-Patienten, Beatmungseinstellung bei ALS). Eine zulässige Behandlungsmaßnahme muss grundsätzlich 2 Voraussetzungen erfüllen:

1. Für den Beginn oder die Fortführung besteht nach Einschätzung der behandelnden Ärzte eine medizinische Indikation.

2. Die Durchführung entspricht dem Patientenwillen.

Im Verlauf der Behandlung können sich die medizinische Indikation oder der Patientenwille ändern.

- Zum einen kann das initiale Therapieziel zunehmend unrealistisch werden aufgrund nicht therapierbarer Pathologien,

- zum anderen kann der Patient seine Zustimmung entziehen.

Dabei heißt die Abkehr vom kurativen Ansatz niemals die Abkehr von einer medizinischen Versorgung. Die Begleitung des Patienten im Sterben ist ärztliche Pflicht und muss genau so akribisch erfolgen wie die Begleitung zurück ins Leben. Doch gerade der Wechsel der Therapierichtung ist oft Gegenstand lebhafter Diskussionen und Grund von Missverständnissen im therapeutischen Team oder zwischen diesem und den Angehörigen. Die Pflicht zur Aufklärung umfasst selbstverständlich auch die Aufklärung zu Maßnahmen am Lebensende. Wichtig ist zu klären, dass die Einstellung medizinisch nicht (mehr) sinnvoller Maßnahmen keine aktive Sterbehilfe ist [2]. Patienten sterben nicht am Entzug der Therapie, sondern an der schweren Grunderkrankung ( $\bullet$ Abb. 1).

\section{Erläuterung}

1. Die Definition des Therapieziels ist sehr wichtig: Vom Extrem der vollen kurativen Therapie bis hin zum kompletten Einstellen aller Maßnahmen sind viele Varianten im Spektrum bzw. Abstufungen möglich. Eine zu feine Abstufung des Therapieausmaßes ist nicht praktikabel und führt zu Unsicherheiten bei allen Beteiligten. Die unten genannten 4 Kategorien sind unserer Erfahrung nach praktikabel. Nach voller Therapie mit allen Optionen schließt sich die erste Einschränkungsstufe an: der Verzicht auf definierte Maßnahmen. Dieser Verzicht auf konkrete Maßnahmen, z. B. eine Reanimation, die Dialyse oder erneute Beatmung, kann trotz kurativen Weiterführens aller anderen Maßnahmen sinnvoll sein. Hier sind als Beispiel auch Zeugen Jehovas zu nennen, bei denen grundsätzlich Intensivmedizin indiziert ist, die Transfusion von Blut und Blutprodukten aber unterbleiben muss. Eine Therapielimitierung („DNR-Code“ [Do Not (attempt to) Resuscitate]) ist NICHT gleichbedeutend mit einem Einstellen der kurativen Therapie. Daher ist eine klare Zielsetzung für jeden im therapeutischen Team notwendig. Unterschieden werden muss auch zwischen aktuellen Tageszielen, den Zielen der akuten Erkrankung und den Zielen der Grunderkrankung. So kann ein Patient mit einer prinzipiell chronischen oder lebenslimitierenden Erkrankung (z.B. Krebserkrankung) mit vollem kurativen Ansatz Intensivtherapie erhalten, um eine vorübergehende Akuterkrankung zu behandeln. Die Stufe des Abbruchs aller kurativen Bemühungen und das Durchführen einer Symptomkontrolle folgen als Nächstes; z.T. können diese Maßnahmen auch bereits parallel begonnen werden. 


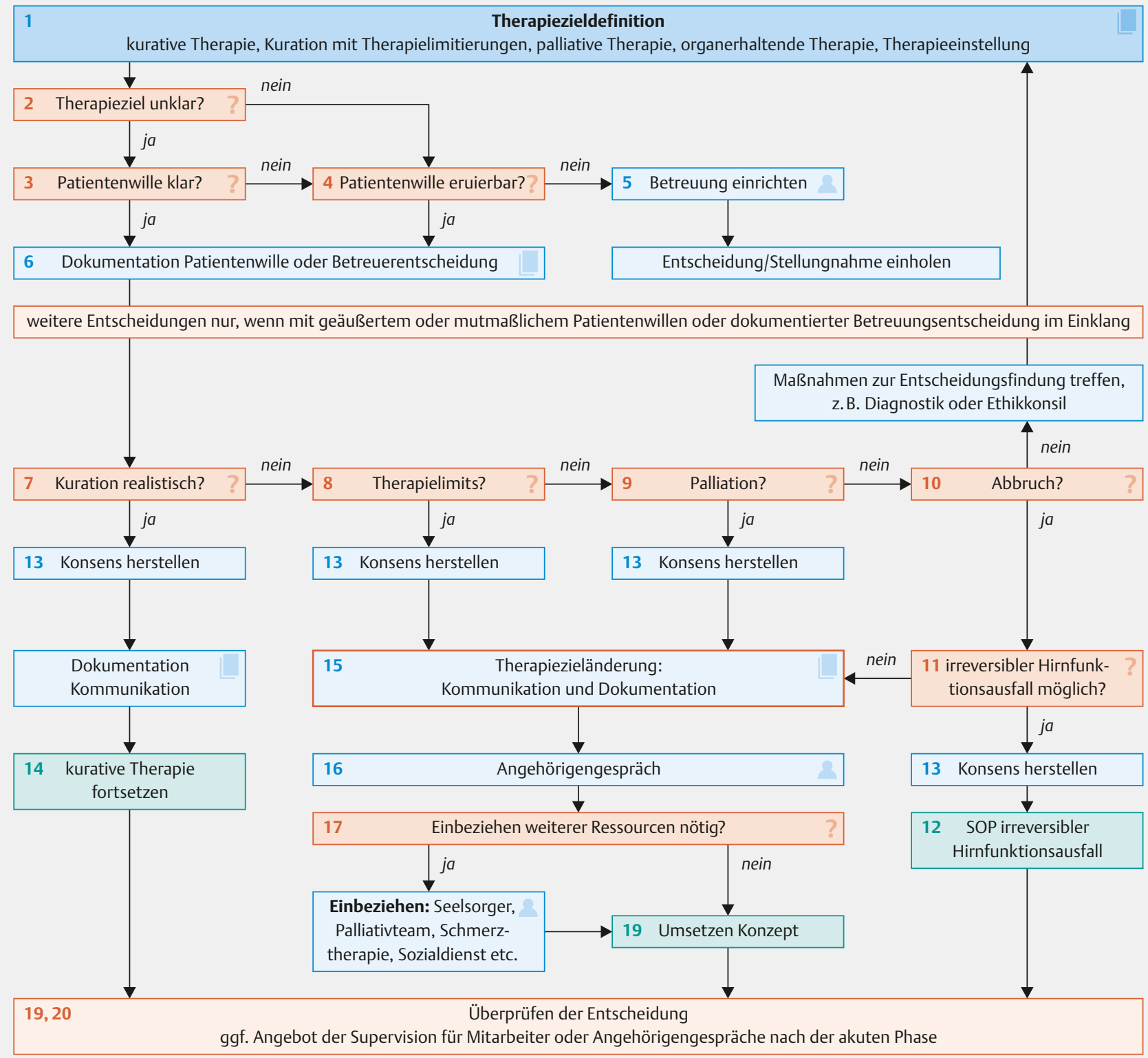

- Abb. 1 SOP Therapiezieländerung. ${ }^{1-20}$ siehe Kapitel „Erläuterung“.

2. Die Frage nach dem Therapieziel ist immer ethischrechtlich geboten; wenn keine Deutlichkeit besteht, muss eine Klärung erzwungen und dokumentiert werden.

3. Der mutmaßliche oder geäußerte Patientenwille ist Grundlage jeder Therapie und muss klar sein. Therapien abweichend von diesem Willen sind strafrechtlich relevant.

4. Kann der Patient seinen Willen nicht selbst äußern, so sind Patientenverfügungen und/oder Betreuungsvollmachten wirksam, die zwingend zu beachten sind [3]. Es sei angemerkt, dass Meinungen sich ändern können, wenn aus einem gesunden Menschen ein Pa- tient wird. Die Einstellung der Therapeuten ist in diesem Zusammenhang irrelevant. Ein dahingesagtes „Ich würde nie mit einer Querschnittslähmung leben wollen" heißt nicht zwingend, dass ein Querschnittsgelähmter nach einem Unfall nun den Abbruch aller therapeutischen Maßnahmen will. Dies macht Entscheidungen oft schwierig [4]. Deshalb sind Angehörigengespräche sehr wichtig, um eine (In-)Kongruenz der aktuelleren Einstellungen des Patienten mit seinen früheren Bekundungen zu erkennen. Sehr wichtig und unbedingt klarzustellen ist den Angehörigen (auch Vorsorgebevollmächtigten) gegenüber, dass es nicht um ihre Einstellung bzw. Einschätzung der Si- 
tuation geht, sondern um die des Patienten. Die Entscheidungsverantwortung über Start oder Stopp einer Therapie fällt nicht der Angehörige, sondern der Arzt im Sinne des Patienten. Dies sollte man in den Gesprächen immer klarstellen, um spätere Schuldgefühle zu vermeiden.

5. Sollten Patientenwille und/oder Zustimmungslage unklar sein, so ist eine gesetzliche Betreuung einzurichten [3]. Im Zweifel muss ein Gericht zu strittigen Entscheidungen befragt werden.

6. Die Dokumentation von Patientenwille und Therapieziel ist entscheidend. Zur Dokumentation von Therapiezielen existiert eine Anregung der DIVI [5]. Zum einen, damit jedes Mitglied des Teams Entscheidungen nachvollziehen und danach handeln kann, zum anderen für die medikolegale Nachprüfbarkeit.

7. Unter Therapiebegrenzung können folgende Vorgehensweisen fallen:

- Verzicht auf zusätzliche kurative Maßnahmen

- Verzicht auf Ausweitung bestehender kurativer Maßnahmen

- Reduktion bestehender kurativer Maßnahmen

- Absetzen (aktives Beenden) bestehender kurativer Maßnahmen

Zur Entscheidung der Kurierbarkeit ist jeder im therapeutischen Team einzubeziehen. Die Therapien sollten im Rahmen von u. a. Visiten diskutiert und nötigenfalls Zusatzdiagnostik zur Klärung durchgeführt werden. Hilfreich sind hier auch oft Teamsitzungen außerhalb der Station, wenn v.a. bei Langzeitpatienten therapeutische Ansätze in den verschiedenen Berufsgruppen auseinanderstreben. Hier können die Berufsgruppen wieder zusammengeführt werden.

8. Die Limitierungen, also Therapieeinstellung oder -begrenzung, können vielfältig sein: vom Verzicht auf zusätzliche invasive Therapien (keine Reanimation/ ECMO, keine Dialyse) über den Verzicht zur Ausweitung bestehender Therapien (z. B. Eskalation einer Katecholamintherapie) bis hin zum Ausschluss bestimmter Versorgungsformen (z. B. keine Rehabilitation, keine Prophylaxemaßnahmen, keine Therapie von Komplikationen) ist alles zu besprechen und zu dokumentieren.

9. Eine Therapiebegrenzung im Rahmen einer palliativen Begleitung kann für den kurzfristigen Verlauf sinnvoll sein (z.B. Morphinperfusor zur Linderung von Atemnot in der Agonie), kann aber auch auf den langfristigen Verlauf ausgerichtet sein (z. B. Einbinden des hausärztlichen Palliativdienstes bei einem Patienten mit metastasiertem Bronchialkarzinom nach überstandener Pneumonie). Wichtig sind:

- menschenwürdige Unterbringung

- menschliche Zuwendung

- Körperpflege

- Lindern von Schmerzen, Luftnot, Übelkeit und anderen subjektiv belastenden Symptomen

- Stillen von subjektiv vorhandenem Hunger und Durst
10. Ein kompletter Abbruch der Therapie ist nur sehr selten indiziert. Meist muss der Patient durch verschiedene Maßnahmen, z.B. durch gezielte palliativmedizinische Interventionen zur Symptomkontrolle oder durch einen Seelsorger, in der letzten Lebensphase begleitet werden. Es gibt auch Situationen, die so ausweglos sind bzw. in denen eine Fortsetzung der Intensivtherapie nicht gewünscht ist, sodass eine palliative Extubation erfolgen sollte.

11. Bei potenziell irreversiblem Hirnfunktionsausfall und der theoretischen Möglichkeit einer Organspende muss ein entsprechendes Protokoll durchgeführt werden.

12. Hierzu verweisen wir auf die SOP Hirntod („irreversibler Hirnfunktionsausfall“) in der vorigen Ausgabe der Intensivmedizin up2date [6, 7].

13. Natürlich muss im therapeutischen Team Konsens hergestellt werden. Visiten- und Besprechungssituationen sind hier genauso geeignet wie Teamsitzungen. Wichtig ist, dass jeder im therapeutischen Team (inkl. der Angehörigen) gehört wird, um sicherzugehen, dass alle Aspekte abgewogen werden. Hier passiert es leicht, dass Chefärzte weniger starke Teammitglieder überrollen. Neben Unmut, Frustration und Gewissensproblemen kann hieraus eine unumkehrbare Fehlentscheidung resultieren. Allerdings darf der Teamgedanke nicht zur Entscheidungslähmung führen. Auch wenn ein Mitglied nicht greifbar ist, müssen die Entscheidungen wie auch andere Therapieentscheidungen getroffen werden, ggf. über Vertreterregelung.

14. Das Fortsetzen der kurativen Therapie wird bei den meisten Patienten in Abwägung aller Befunde durchgeführt.

15. Ändert sich das Ziel, so sind die Kommunikation im Team und die Dokumentation in der Akte besonders wichtig. Es sei angemerkt, dass auch eine Therapielimitierung bei einem günstigen Verlauf wieder in eine volle kurative Therapie geändert werden kann.

16. Ein dokumentiertes und strukturiertes Angehörigengespräch inkl. eines Hilfsangebots (z.B. Seelsorge, Anrufen von Freunden oder weiteren Familienangehörigen, Hausarzt) ist Pflicht des behandelnden Arztes.

17. Das Begleiten von Patienten am Lebensende erfordert Expertise. Hier können weitere Ressourcen (z. B. Seelsorge, Musiktherapie, Sozialdienst, Pflegedienste) genutzt werden. Zu beachten ist die Versorgungsrealität, wenn z. B. der Patient zurück nach Hause soll. Auch für Hinterbliebene gilt es zu sorgen.

18. Natürlich muss im kurativen Therapieansatz das Therapiekonzept umgesetzt werden. Aber auch bei Therapiebegrenzung sollte es Regelungen geben. In jedem Haus sollte ein End-of-Life-Konzept vorliegen. Der Umgang mit Sterbenden und Verstorbenen muss geregelt sein. Neben medikamentösen und nicht medikamentösen Therapien zur Linderung von Leid 
(Schmerz, Hunger, Durst, Angst, Luftnot) sollten auch ein möglichst würdevoller Rahmen (trotz Intensivstation) und spirituelle Aspekte in Betracht kommen. Diese sind vor dem Hintergrund des Kulturkreises sehr wichtig. Auch hier sei aber angemerkt, dass die Spiritualität des Behandlungsteams nicht Maß der Dinge ist, sondern der Patient und sein Bedürfnis im Mittelpunkt stehen.

19. Die Entscheidungen müssen ständig reevaluiert werden. Eine Entscheidung kann im Verlauf bei Auftreten neuer Gegebenheiten revidiert werden. Das Begleiten von Menschen am Lebensende ist oft auch für Mitglieder des therapeutischen Teams belastend. Angebote zur Supervision oder einfach nur ein Gespräch unter Kollegen ist sinnvoll.

20. Oft hilft es Angehörigen, wenn sie das Angebot erhalten, nach einigen Wochen noch mal mit dem Intensivmediziner reden zu können, Fragen zu klären und Gedanken auszutauschen. Eine Begleitung der Hinterbliebenen in ihrer Trauer, ein Umgehen mit den Erfahrungen, Ängsten und vielleicht auch Vorwürfen ist essenziell. Leider wird dies im Klinikalltag oftmals vergessen. Spezifische, professionelle Anlaufstellen sind selten.

\section{Interessenkonflikt}

Die Autoren geben an, dass kein Interessenkonflikt besteht.

\section{Autorinnen / Autoren}

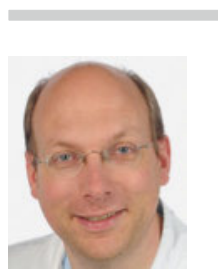

\section{Björn Ellger}

Prof. Dr. med. Direktor der Kliniken für Anästhesiologie, Intensivmedizin und Schmerztherapie, Klinikum Westfalen, Dortmund.

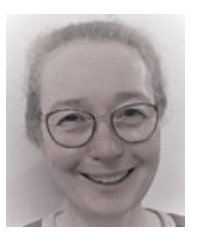

\section{Corinna Kelbel}

Dr. med. Oberärztin an der Klinik für Anästhesiologie, Intensivmedizin und Schmerztherapie, Klinikum Westfalen, Standort Klinik am Park, Lünen.

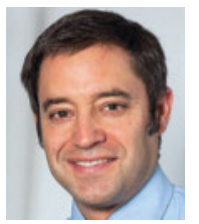

\section{Julian Bösel}

Prof. Dr. med. FNCS. Direktor der Klinik für Neurologie am Klinikum Kassel.

\section{Korrespondenzadresse}

Prof. Dr. med. Björn Ellger

Kliniken für Anästhesiologie, Intensivmedizin

und Schmerztherapie

Klinikum Westfalen GmbH

Am Knappschaftskrankenhaus 1

44309 Dortmund

bjoern.ellger@klinikum-westfalen.de

Literatur

[1] Jansen U, Burchardi H, Duttge G et al. Therapiezieländerung und Therapiebegrenzung in der Intensivmedizin - Positionspapier der Sektion Ethik der DIVI. Im Internet: http://www.divi.de/images/Dokumente/Empfehlungen/Therapiezielaenderung/Positionspapier_Ethik_2012.pdf; Stand: 09.10.2017

[2] Reith S, Janssens U. Dying with/despite a pacemaker. Med Klin Intensivmed Notfmed 2014; 109: 19-26. doi:10.1007/ s00063-013-0282-7

[3] Bundesärztekammer. Empfehlungen der Bundesärztekammer und der Zentralen Ethikkommission bei der Bundesärztekammer zum Umgang mit Vorsorgevollmacht und Patientenverfügung in der ärztlichen Praxis. Im Internet: http://www.bundesaerztekammer.de/fileadmin/user_upload/downloads/Patientenverfuegung_und_Vollmacht_Empfehlungen_BAeK-ZEKO_DAe1.pdf; Stand: 09.10.2017

[4] Gruß M, Salomon F. Autonomie und Fürsorge in der Intensivmedizin - Praktisches Vorgehen in schwierigen Situationen. Anästhesist 2016; 65: 875-888

[5] Nietzke G, Böll B, Burchardi B et al. Dokumentation der Therapiebegrenzung - Empfehlung der Sektion Ethik der Deutschen Interdisziplinären Vereinigung für Intensiv- und Notfallmedizin (DIVI) unter Mitarbeit der Sektion Ethik der Deutschen Gesellschaft für Internistische Intensivmedizin und Notfallmedizin (DGIIN). Med Klin Intensivmed Notfmed 2017. doi:10.1007/ s00063-017-0321-x

[6] Bösel J, Ellger B. SOP Hirntod (“irreversibler Hirnfunktionsausfall”). Intensivmed up2date 2017; 13: 245-248

[7] Bundesärztekammer. Arbeitspapier zum Verhältnis von Patientenverfügung und Organspendeerklärung. Im Internet: http://www.bundesaerztekammer.de/fileadmin/user_upload/ downloads/Arbeitspapier_Patientenverfuegung_Organspende_18012013.pdf; Stand: 09.10.2017

\section{Bibliografie}

DOI https://doi.org/10.1055/s-0043-119369

Intensivmedizin up2date 2017; 13: 351-354

(c) Georg Thieme Verlag KG Stuttgart · New York ISSN 1614-4856 